\title{
Fault tolerant control using an admissible model matching approach
}

\author{
M. Staroswiecki \\ Ecole Polytechnique Universitaire de Lille \\ University of Lille I \\ 59655 Villeneuve d'Ascq cedex
}

\begin{abstract}
In the pseudo-inverse method (PIM) the Frobenius norm based distance between the closed loop model of the faulty system and some reference model is minimized. Stability issues are considered in the Modified PIM (MPIM). This paper proposes to use a set of admissible models, rather than searching for an optimal one which does not provide any stability / performance guarantee. The approach allows to characterize the set of recoverable faults, and to associate some robustness property with the fault adaptation scheme.
\end{abstract}

\section{INTRODUCTION}

The Fault Tolerant Control problem [1], [8] has been addressed considering many different objectives : stability, disturbance attenuation [6], model matching [3], [4], predictive control [5], optimal control [9], [10].

Model matching and the pseudo-inverse method (PIM) have been first introduced in flight control systems, see e.g. [2], [3], [7], to deal with situations where pilots must keep faulty systems at hand. The PIM rationale is to redesign the control of the faulty system, in such a way that some distance between the nominal and the accommodated closed loop systems is minimized. The considered distance is the Frobenius norm of the difference between the state space matrices associated with the nominal and the accommodated system behavior. However, there are major drawbacks to the standard PIM : the stability of the solution is not guaranteed (a problem which has been addressed by [3]) and moreover, the rationale appears to be quite weak.

In a previous paper [11], the model matching problem was revisited by searching for the solution within an admissible set of reference models, instead of finding the best approximation of an ideal one. This paper extends the previous results by considering a more versatile (but more complex) definition of the admissible set of reference models, which leads to the consideration of a multi-criteria optimization problem. In addition, it shows that the approach enjoys a robustness property by which it combines both active and passive fault tolerance features.

It is organized as follows. Section 2 presents the nominal control problem. Section 3 addresses both the fault accommodation and the system reconfiguration strategies to solve the fault tolerant control problem. In Section 4 the classical PIM method, which is used when the problem has no exact solution, is recalled and its rationale is discussed. Section 5 proposes a more practical point of view associated with the definition of an admissible set of models that can be matched. In Section 6, two dual approaches are presented for the determination of the set of faults that are recoverable, and the robustness property is discussed in Section 7. The simple example provided in [3] is used in order to illustrate the approach.

\section{THE MODEL MATCHING PROBLEM}

Let subscript $n$ stand for nominal. In the LTI model matching problem, the objective $O_{n}$ is to design a control law for the system described by the constraints

$$
C_{n}: \dot{x}(t)=A_{n} x(t)+B_{n} u(t)
$$

such that the closed loop behavior follows the reference model

$$
\dot{x}(t)=M^{*} x(t)+N^{*} e(t)
$$

where $x(t) \in R^{n}$ is the system state, $u(t) \in R^{m}$ is the control vector, the pair $M^{*}, N^{*}$ is given ( $M^{*}$ is obviously chosen to be stable), and $e(t) \in R^{q}$ is an arbitrary input vector. If state feedback is considered, the set of controls is

$$
U_{n}:\left\{\begin{aligned}
R^{n} \times R^{q} & \longrightarrow R^{m} \\
(x(t), e(t)) & \longmapsto u(t)=G_{n} e(t)-K_{n} x(t)
\end{aligned}\right.
$$

where $G_{n}$ and $K_{n}$ are matrices to be determined. The nominal solution of this problem is obtained by solving the system

$$
\begin{aligned}
A_{n}-B_{n} K_{n} & =M^{*} \\
B_{n} G_{n} & =N^{*}
\end{aligned}
$$

for $K_{n}$ and $G_{n}$, and a necessary and sufficient condition for solutions to exist is that

$$
\begin{aligned}
\operatorname{Im}\left(A_{n}-M^{*}\right) & \subseteq \operatorname{Im}\left(B_{n}\right) \\
\operatorname{Im}\left(N^{*}\right) & \subseteq \operatorname{Im}\left(B_{n}\right)
\end{aligned}
$$

Assuming that $\operatorname{rank}\left(B_{n}\right)=m$, the unique solution is given by

$$
\begin{aligned}
& K_{n}=B_{n}^{+}\left(A_{n}-M^{*}\right) \\
& G_{n}=B_{n}^{+} N^{*}
\end{aligned}
$$

where $B_{n}^{+}$is the left pseudo-inverse of $B_{n}$, i.e. a matrix such that $B_{n}^{+} B_{n}=I$.

Definition 1 (Feasibility): For a given pair $\left(A_{n}, B_{n}\right)$ matrices $M^{*}$ (resp. $N^{*}$ ) which satisfy the compatibility conditions (3) are called feasible, and their set is noted $\mathcal{M}\left(A_{n}, B_{n}\right)$ (resp. $\left.\mathcal{N}\left(A_{n}, B_{n}\right)\right)$. 


\section{FAult Tolerant CONTROL}

The fault tolerant control problem is defined by the triple $<O_{n}, C_{f}, U_{f}>$, where $O_{n}$ is the (unchanged) objective, and $C_{f}$ (resp. $U_{f}$ ) is the set of constraints (resp. of admissible controls) associated with the post-fault system to be controlled.

$$
\begin{array}{ll}
O_{n}: & \dot{x}(t)=M^{*} x(t)+N^{*} e(t) \\
C_{f}: & \dot{x}(t)=A_{f} x(t)+B_{f} u(t) \\
U_{f}: & u(t)=G_{f} e(t)-K_{f} x(t)
\end{array}
$$

The post-fault system may result from system reconfiguration, i.e. the faulty components are switched-off and healthy ones may be switched-on. In that case, its model $\left(A_{f}, B_{f}\right)$ is known since the models of all healthy components are known. In the reconfiguration strategy, the FDI system is obviously required only to detect the occurrence of a fault and to isolate the faulty components (so as to switch them off).

Fault accommodation (instead of system reconfiguration), is the strategy where the post-fault system is the faulty one. Therefore $\left(A_{f}, B_{f}\right)$ is the model of the faulty system (assumed to be still linear) and the FDI algorithm is not only required to detect and isolate faults, but also to identify the faulty system model.

In both cases, the pair $\left(A_{f}, B_{f}\right)$ may - or not - allow a solution to exist for the model matching problem.

Definition 2 (Consistent system): The post-fault system $\left(A_{f}, B_{f}\right)$ is consistent if and only if it allows to achieve the control objective, which means that matrices $M^{*}$ and $N^{*}$ are still feasible : $M^{*} \in \mathcal{M}\left(A_{f}, B_{f}\right)$ and $N^{*} \in \mathcal{N}\left(A_{f}, B_{f}\right)$.

The new control law $\left(K_{f}, G_{f}\right)$ can be found, by applying (4) with entries $\left(A_{f}, B_{f}\right)$ instead of $\left(A_{n}, B_{n}\right)$, if and only if the pair $\left(A_{f}, B_{f}\right)$ is consistent.

Definition 3 (Recoverable fault): A fault is recoverable by a given strategy (system reconfiguration or fault accommodation), or it is admissible for this strategy, if and only if the associated post-fault system $\left(A_{f}, B_{f}\right)$ is consistent.

\section{ApPRoximate MODEL MATCHING}

When the pair $\left(A_{f}, B_{f}\right)$ is not consistent, neither accommodation nor reconfiguration can provide a solution. Strictly speaking, the objective is not tolerant to such faults. However, the exact requirement may seem too demanding, and approximate rather than exact solutions are of interest. The Pseudo-inverse method (PIM) sets a "best matching" objective instead of an "exact matching" one. This method is recalled and commented in this section. For the sake of conciseness, only the problem associated with matrices $A, B, K$ and $M$ is considered, since results for $N$ and $G$ are quite similar.

\section{A. The Pseudo-Inverse Method}

The approximate model matching problem was first stated in [4]. When the closed loop matrix $A_{f}-B_{f} K_{f}$ cannot be made equal to $M^{*}$, approximate solutions are computed, which minimize the criterion $J=\left\|A_{f}-B_{f} K_{f}-M^{*}\right\|_{F}^{2}$ where $\|P\|_{F}$ is the Frobenius norm of matrix $P$,

$$
\|P\|_{F}^{2}=\sum_{i, j} p_{i j}^{2}
$$

Simple calculations show that the control law $K_{f}$ which minimizes $J$ is still given by (4), hence the name PseudoInverse. Replacing $K_{f}$ by its optimal value, the result is

$$
A_{f}-B_{f} K_{f}-M^{*}=\left(I-B_{f} B_{f}^{+}\right)\left(A_{f}-M^{*}\right)
$$

which leads to the value

$$
J=\sum_{i=1}^{n}\left(a_{f}^{i}-m^{* i}\right)^{\tau}\left(I-B_{f} B_{f}^{+}\right)\left(a_{f}^{i}-m^{* i}\right)
$$

where $a_{f}^{i}$ and $m^{* i}$ are respectively the $i^{t h}$ columns of $A_{f}$ and $M^{*}$. Note that $J^{*}$ is zero when the pair $\left(A_{f}, B_{f}\right)$ is consistent, but otherwise it is non-zero. There are several major drawbacks to the standard PIM, which are now commented.

\section{B. Stability}

Exhibiting the closed loop behavior nearest to the reference one, does not guarantee the accommodated/reconfigured system to be stable. Extensions have been proposed in the literature, using constrained optimization, namely the criterion $J$ is minimized under the constraint that $A_{f}-B_{f} K_{f}$ is stable [3]. However, the constrained optimization problem is based on sufficient stability conditions (it may therefore provide very conservative solutions), and it may be complex to solve in real time.

\section{PIM rationale}

The rationale of the approach is questionable since it does not address several important points.

1) Finding the accommodated/reconfigured system closest to the reference one, does not guarantee that it will be close enough to exhibit a satisfactory dynamic behavior (even when it is stable).

2) Moreover, the very meaning of "closest" is questionable, since the choice of the Frobenius norm based distance is arbitrary. Any distance can be used when the problem is to prove the equality of two models, but in the approximate model matching problem, choosing another distance would obviously provide another solution.

3) It follows from the problem setting that any fault $\left(A_{f}, B_{f}\right)$ can be recovered, since there is always a solution to the minimal distance problem. This is indeed a point that contradicts our feeling that in some fault situations, there is no accommodated/reconfigured control which achieves satisfactory approximation of $M^{*}$.

4) Nominal and faulty system models are generally uncertain, however robustness considerations are not included in the PIM problem setting. 


\section{ADMISSIBLE MODEL MATCHING}

In the admissible model matching approach, we consider a set of system matrices that are acceptable, instead of considering one single reference model and try to best approximate it [11]. Let $\mathcal{M}$ be a set of matrices such that any solution of

$$
\dot{x}(t)=M x(t), \forall M \in \mathcal{M}
$$

has acceptable dynamic behavior. The set of reference models $\mathcal{M}$ is defined off-line, and it obviously contains only stable matrices. We assume that the set of reference models is specified by a set of $d$ inequality constraints

$$
\mathcal{M}=\left\{M \text { s.t. } \Phi\left(m_{i j}, i=1, . . n, j=1, . . n\right) \leq 0\right\}
$$

where $m_{i j}, i=1, . . n, j=1, . . n$ are the entries of matrix $M, \Phi: R^{n \times n} \rightarrow R^{d}$ is a given vector function, and the constraints are written $\Phi(M)$ for short.

\section{A. Example}

The LTI system

$$
\left(A_{n}, B_{n}\right)=\left(\left(\begin{array}{cc}
-1 & 0 \\
0 & -1
\end{array}\right),\left(\begin{array}{l}
1 \\
5
\end{array}\right)\right)
$$

was used as an illustration in [3], where the reference model was $\dot{x}(t)=M^{*} x(t)$ with

$$
M^{*}=\left(\begin{array}{cc}
-2 & 0 \\
-5 & -1
\end{array}\right)
$$

The closed loop control

$$
u=-x_{1}
$$

achieves perfect model matching, with closed loop system poles $\lambda_{1}=-1$ and $\lambda_{2}=-2$. In [3] the fault

$$
\left(A_{f}, B_{f}\right)=\left(\left(\begin{array}{cc}
-1 & 0 \\
0 & -1
\end{array}\right),\left(\begin{array}{c}
-1 \\
1
\end{array}\right)\right)
$$

was considered. The pair $\left(A_{f}, B_{f}\right)$ being not consistent with $M^{*}$, exact model matching is not possible; the PIM provides the closed loop control

$$
u=-2 x_{1}
$$

which can easily be checked to cause an unstable closed loop behavior. The modified approach proposed in [3] gives

$$
u=-0.8 x_{1}
$$

which results in the closed loop matrix

$$
\left(\begin{array}{cc}
-0.2 & 0 \\
-0.8 & -1
\end{array}\right)
$$

with poles -1 and -0.2 . Note that this approach indeed allows to stabilize the system but offers no real control over the behavior of the closed loop system which results from fault accommodation : the nearest closed loop matrix (under stability constraints) is selected, which in this example results in one unchanged pole, and the other one divided by a factor 10.
Following the admissible model matching approach, define the set of admissible closed loop matrices by

$$
\begin{aligned}
& \mathcal{M}=\left\{\left(\begin{array}{ll}
p & q \\
r & s
\end{array}\right): T \text { is true }\right\} \\
& T:\left\{\begin{array}{l}
\Phi_{1}(M) \triangleq 2 p^{2}+2 s^{2}-5 p s+9 r q=0 \\
\Phi_{2}(M) \triangleq p+s+2,7 \leq 0 \\
\Phi_{3}(M) \triangleq-p-s-3.3 \leq 0
\end{array}\right.
\end{aligned}
$$

It can easily be checked that any admissible matrix in $\mathcal{M}$ is defined by four inequality constraints $\left(\Phi_{1}(M) \leq 0\right.$, $-\Phi_{1}(M) \leq 0, \Phi_{2}(M) \leq 0$ and $\Phi_{3}(M) \leq 0$ ) and has eigenvalues $\tau \lambda_{1}$ and $\tau \lambda_{2}$ with $\tau \in[0.9,1.1]$. This means that, instead of trying to match the reference model $M^{*}$, the designer tries to obtain an admissible closed loop matrix, such that its eigenvalues lie within a $\pm 10 \%$ range of the eigenvalues of $M^{*}$.

\section{B. Problem definition}

The admissible model matching problem is defined by the triple

$$
\begin{array}{cc}
O_{i}: & A_{i}-B_{i} K_{i} \in \mathcal{M} \\
C_{i}: & \dot{x}(t)=A_{i} x(t)+B_{i} u(t) \\
U_{i}: & u(t)=-K_{i} x(t)
\end{array}
$$

where index $i=n$ in nominal operation, and $i=f$ in faulty operation. The problem is to find state feedback control laws $u(t)=-K_{i} x(t)$ such that the closed loop systems

$$
\dot{x}(t)=\left(A_{i}-B_{i} K_{i}\right) x(t)
$$

satisfy $A_{i}-B_{i} K_{i}=M_{i}$ for some $M_{i} \in \mathcal{M}$, whatever the value of index $i \in\{n, f\}$.

\section{Existence of solutions}

From (6) it is seen that nominal and faulty situations both lead to the same problem to be solved. Let $(A, B)$ be a system model - $\left(A_{n}, B_{n}\right)$ when nominal, $\left(A_{f}, B_{f}\right)$ when faulty - the problem consists of finding a matrix $K$ which satisfies

$$
\exists M \in \mathcal{M}: A-B K=M
$$

From the definitions of $\mathcal{M}$ and $\mathcal{M}(A, B)$ it follows that a necessary and sufficient condition for the existence of a solution is

$$
\mathcal{M}(A, B) \cap \mathcal{M} \neq \varnothing
$$

The definition of a consistent system has therefore to be extended : $(A, B)$ is consistent if and only if it satisfies (7). For any consistent system $(A, B)$ there is a set of solutions to the admissible control problem, given by

$$
\begin{gathered}
\mathcal{K}(A, B)=\left\{K: K=B^{+}(A-M), \ldots\right. \\
\ldots M \in \mathcal{M}(A, B) \cap \mathcal{M}\}
\end{gathered}
$$




\section{Characterization OF RECOVERABle FAUlts}

Recoverable faults are such that the pair $(A, B)$ is consistent, which means that the set of solutions $\mathcal{K}(A, B)$ is not empty. In practice, it is enough to find a matrix $M$ such that

$$
\begin{aligned}
\Phi(M) & \leq 0 \\
\left(I-B B^{+}\right)(A-M) & =0
\end{aligned}
$$

since the second condition insures that it is feasible. By writing this condition under the form

$$
J(M)=\sum_{i=1}^{n}\left(a^{i}-m^{i}\right)^{\tau}\left(I-B B^{+}\right)\left(a^{i}-m^{i}\right)=0
$$

dual solution approaches are suggested as follows :

- Problem 1 : solve the constrained optimization problem

$$
\min _{M} J(M) \text { under } \Phi(M) \leq 0
$$

- Problem 2 : solve the multicriteria optimization problem

$$
\min _{M} \Phi(M) \text { under } J(M)=0
$$

\section{A. Problem 1}

Problem 1 is a classical quadratic optimization problem under inequality constraints, for which algorithms are available in the literature. Note that there is here no drawback in using the Frobenius norm in the problem definition. Indeed, the norm is not interpreted as the distance between two systems (as in the PIM), but it is only used to find (when it exists) an admissible matrix $(M \in \mathcal{M})$ which is also feasible $(M \in \mathcal{M}(A, B))$. When a solution exists, it will be found whatever the norm which is used. A solution will not exist when faults are not recoverable.

Theorem 4: Let $M^{*}$ be a solution of Problem 1. A necessary and sufficient condition for the admissible model matching problem to have a solution for system $(A, B)$ is that

$$
J\left(M^{*}\right)=0
$$

Proof: The proof is evident, from the definition of $\mathcal{M}$ and the fact that

$$
J\left(M^{*}\right)=0 \Longleftrightarrow M \in \mathcal{M}(A, B)
$$

\section{B. Problem 2}

Substituting for the constraint, Problem 2 can be rewritten as a non constrained (but multicriteria) optimization problem

$$
\min _{K} \Phi(A-B K)
$$

As vector optimization is invoked, let us recall the definition of the domination relation.

Definition 5: Let $V$ be some decision set and let $\Psi$ : $V \rightarrow R^{d}$ be a vector function that associates an outcome $\Psi(v) \in R^{d}$ with any decision $v \in V$. Decision $v$ dominates decision $w(v \succeq w)$ if and only if $\Psi(v) \leq \Psi(w)$ - which means that $\Psi_{i}(v) \leq \Psi_{i}(w)$ for all components $i=1, \ldots d$; decision $v$ strictly dominates decision $w(v \succ w)$ if and only if $\Psi(v) \leq \Psi(w)$ and $\Psi(v) \neq \Psi(w)$.

Let $\mathcal{P}(A, B)$ be the set of Pareto-optimal solutions of problem (10), i.e. the set of matrices $K^{*}$ such that (11) and (12) are true :

$$
\begin{gathered}
\forall K \notin \mathcal{P}(A, B), \exists K^{*} \in \mathcal{P}(A, B) \text { such that } K^{*} \succ K \\
\left\{\begin{array}{c}
\forall K_{1}^{*} \in \mathcal{P}(A, B) \text { and } \forall K_{2}^{*} \in \mathcal{P}(A, B) \\
K_{1}^{*} \succeq K_{2}^{*} \Longrightarrow K_{2}^{*} \succeq K_{1}^{*}
\end{array}\right.
\end{gathered}
$$

Property (11) means that whatever the non Pareto-optimal matrix $K$, there is a Pareto-optimal one, $K^{*}$ that strictly dominates it, being better at least for one and worse for none of the criteria. Property (12) means that Pareto-optimal solutions are not comparable (should $K_{1}^{*}$ dominate $K_{2}^{*}$ then $K_{2}^{*}$ dominates $K_{1}^{*}$ and therefore they are equivalent : no Pareto-optimal solution can be preferred to another Paretooptimal solution). Algorithms for the efficient computation of Pareto-optimal solutions are available in the multicriteria optimization literature, see e.g. [12], [13].

Theorem 6: A necessary and sufficient condition for the admissible model matching problem to have a solution for the system $(A, B)$ is that the set of Pareto-optimal values of the problem (10) intersects the negative cone in the $\Phi$-space

$$
\exists K \in \mathcal{P}(A, B) \text { such that } \Phi(A-B K) \leq 0
$$

Proof: Sufficiency is evident : let $K^{*}$ satisfy (13), then it is a solution to the problem. Necessity follows from the definition of Pareto-optimal solutions : let $K$ be a solution of the admissible model matching problem. Then, $\Phi(A-$ $B K) \leq 0$ holds true. If $K$ belongs to $\mathcal{P}(A, B)$, then the first statement of (13) is obviously true. If $K$ does not belong to $\mathcal{P}(A, B)$, then there is a matrix $K^{*} \in \mathcal{P}(A, B)$ such that $\Phi\left(A-B K^{*}\right) \leq \Phi(A-B K)$ and $\Phi\left(A-B K^{*}\right) \neq$ $\Phi(A-B K)$. Since $\Phi(A-B K) \leq 0$ the statement is true again.

\section{Example (continued)}

Applying the solution approach of Problem 2, one obtains

$$
\begin{aligned}
& \Phi_{1}(.)=2 k_{1}^{2}+2 k_{2}^{2}-4 k_{1} k_{2}+k_{1}-k_{2}-1=0 \\
& \Phi_{2}(.)=k_{1}-k_{2}+0.7 \leq 0 \\
& \Phi_{3}(.)=-k_{1}+k_{2}-1,3 \leq 0
\end{aligned}
$$

where $\Phi_{i}($.$) stands for \Phi_{i}\left(A_{f}-B_{f} K\right)$. Since the set of Pareto-optimal solutions (shown on Fig.1) is such that

$$
\begin{aligned}
\Phi_{1}\left(A_{f}-B_{f} K\right) & =0 \\
\Phi_{2}\left(A_{f}-B_{f} K\right)+\Phi_{3}\left(A_{f}-B_{f} K\right) & =-0.6
\end{aligned}
$$

it is concluded that the fault is recoverable. The solution is very easily found to be the family of feedback matrices $\left(k_{1}, k_{2}\right)=(k, 1+k), \forall k \in R$.

\section{ROBUSTNESS OF SOLUTIONS}

Let the pair $(A, B)$ and the feedback control $K$ describe the currently operating system, resulting in a closed loop control $M=A-B K$ which is admissible. 


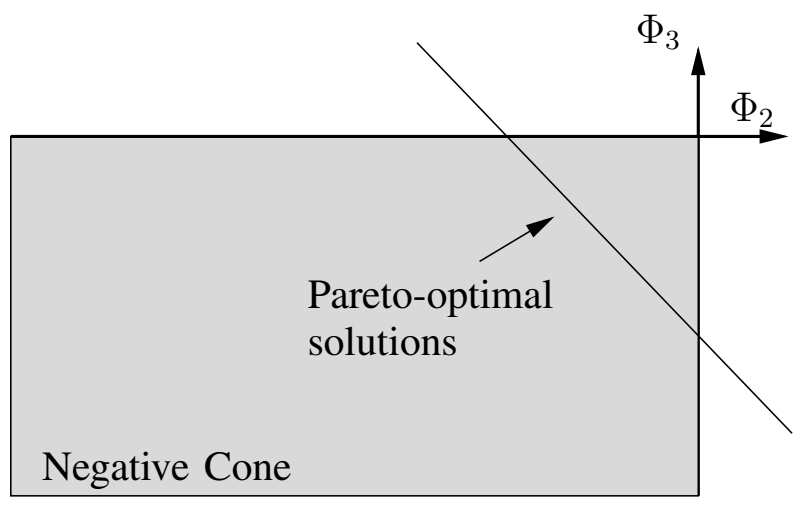

Fig. 1. The set of Pareto-optimal solutions intersects the negative cone

\section{A. Passive fault tolerance property}

Assume that due to the occurrence of a fault, the pair $(A, B)$ is changed into $\left(A^{\prime}, B^{\prime}\right)$, and that $K \in \mathcal{K}\left(A^{\prime}, B^{\prime}\right)$. Then, the closed loop control $K$ is still acceptable, and nothing is to be done in response to the fault.

Given a feedback $K$, the set of all pairs $\left(A^{\prime}, B^{\prime}\right)$ such that $K \in \mathcal{K}\left(A^{\prime}, B^{\prime}\right)$ is the set of all systems for which the closed-loop control $K$ is admissible. Let

$$
\mathcal{A B}(K)=\left\{\left(A^{\prime}, B^{\prime}\right): A^{\prime}-B^{\prime} K \in \mathcal{M}\right\}
$$

be that set. It follows that when changes in the pair $(A, B)$ occur, that are the result of faults, passive fault tolerant control is achieved by the feedback control law $K$, as long as system $\left(A^{\prime}, B^{\prime}\right)$ remains in $\mathcal{A B}(K)$.

\section{B. Robustness degrees}

It may be interesting to evaluate the passive fault tolerance of system $(A, B)$ under the control law $K$. Let $S=$ $(A, B), S^{\prime}=\left(A^{\prime}, B^{\prime}\right)$ and $d\left(S, S^{\prime}\right)$ be some distance defined between systems $S$ and $S^{\prime}$. A natural robustness measure is provided by the indexes

$$
\begin{aligned}
\delta_{m}(S, K) & =\inf _{S^{\prime} \notin \mathcal{A} \mathcal{B}(K)} d\left(S, S^{\prime}\right) \\
\delta_{M}(S, K) & =\sup _{S^{\prime} \in \mathcal{A} \mathcal{B}(K)} d\left(S, S^{\prime}\right)
\end{aligned}
$$

whose interpretation is as follows :

- $\delta_{m}(S, K)$ is the radius of a ball in the systems space, centered on system $S$, such that the control law $K$ is admissible for any system within that ball.

- $\delta_{M}(S, K)$ is the radius of a ball in the systems space, centered on system $S$, such that the control law $K$ is not admissible, whatever the system outside that ball.

In practical situations, the concept of distances between systems has very simple interpretations. For example, if only actuator faults are considered, different systems (e.g. healthy and faulty) are associated with different $B$ matrices. Considering the very standard problem of loss of efficiency in actuator $\mathrm{n}^{\circ} i$, for example, the healthy and faulty situations only differ by column $B_{i}$ of matrix $B$ being multiplied by some factor $\tau_{i}<1$, and the distance between the two systems can obviously be taken as any appropriate function of the quantity $1-\tau_{i}$.

\section{Application to biased fault estimation}

Under the fault accommodation strategy, the model of the faulty system must be identified in real time. Since in practice it will never be perfectly known, the problem is to evaluate the extent to which a problem solution $K_{f}$, elaborated using a fault estimate $\left(A_{f}, B_{f}\right)$, is still valid for the actual fault, say $\left(A_{f}^{\prime}, B_{f}^{\prime}\right)$. This is easily done using the previous remark about the passive fault tolerance property of solutions : the system behavior is satisfactory for any actual fault such that

$$
\left(A_{f}^{\prime}, B_{f}^{\prime}\right) \in \mathcal{A B}\left(K_{f}\right)
$$

It follows that the set of accommodable faults depends (1) on the quality of the fault estimation procedure, and (2) on the choice of the solution $K_{f}$ among the set of all possible solutions (which are all equivalent when the estimation is perfect). For a given choice of $K_{f}$, a upper bound of the estimation error up to which robustness can be guaranteed is given by the following proposition.

Proposition 7: Let $S_{f}^{\prime}=\left(A_{f}^{\prime}, B_{f}^{\prime}\right)$ be a faulty system, $S_{f}=\left(A_{f}, B_{f}\right)$ its estimation provided by the FDI level, and $K_{f} \in \mathcal{K}\left(A_{f}, B_{f}\right)$ the accommodated control selected by the FTC level. A sufficient condition for the faulty system to have an admissible behavior under control law $K_{f}$ is that the estimation error is bounded by

$$
d\left(S_{f}, S_{f}^{\prime}\right) \leq \delta_{m}\left(S_{f}, K_{f}\right)
$$

Proof: In that case, the actual system $S_{f}^{\prime}$ is guaranteed to lie within the ball centered on $S_{f}$ which contains all systems with admissible behaviors when the feedback control law $K_{f}$ is used.

This result immediately suggests an optimization procedure for the choice of the solution $K_{f} \in \mathcal{K}\left(A_{f}, B_{f}\right)$, namely the selected feedback law should allow as much uncertainty as possible for the fault estimation procedure, leading to the problem

$$
K_{f}^{o p t}\left(A_{f}, B_{f}\right)=\arg \max _{K \in \mathcal{K}\left(A_{f}, B_{f}\right)} \delta_{m}\left(S_{f}, K\right)
$$

\section{Example (continued)}

Assume that only the first state variable being measured, the accommodated feedback has been chosen as $\left(\begin{array}{ll}k_{1} & k_{2}\end{array}\right)=\left(\begin{array}{ll}-1 & 0\end{array}\right)$. Then, although the fault tolerant control has been designed for the fault estimate $B_{f}=$ $(-1,1)^{\tau}$ it shows a passive fault tolerance property since it can be verified that it actually accommodates all actuator faults of the form $B_{f}=(-1, \beta)^{\tau}$. Indeed in that case, the closed loop matrix is

$$
\left(\begin{array}{cc}
-2 & 0 \\
\beta & -1
\end{array}\right)
$$

which satisfies the admissibility conditions. It follows that uncertainties on the estimation of the second component of the fault vector $B_{f}$ are not critical. 


\section{CONCLUSION}

Fault tolerance is the property that a system remains able to achieve a given objective (or enjoy a given property) in the presence of faults from a given fault set. In this paper, the model matching objective has been addressed, in the presence of parametric faults such that the system can still be described by a LTI model.

It is proposed to extend the classical and the modified pseudo-inverse methods, by using a set of admissible models, rather than searching for an optimal one which does not provide any guarantee about the post-fault system behavior. This approach applies to both the fault accommodation and the system reconfiguration strategies.

Two necessary and sufficient conditions have been given, which allow to characterize the set of faults that can be recovered. Unlike the PIM or the modified PIM approaches, the approach proposed in this paper exhibits a robustness property by which it combines both passive and active fault tolerance features.

\section{REFERENCES}

[1] Blanke M., M. Kinnaert, J. Lunze and M. Staroswiecki (2003) Diagnosis and Fault Tolerant Control. Springer-Verlag, ISBN 3-54001056-4.

[2] Caglayan A. K., Allen S. M. and K. Wehmuller (1988). Evaluation of a second generation reconfiguration strategy for aircraft flight control systems subjected to actuator failure surface damage. In Proc. of the 1988 IEEE National Aerospace and Electronics Conference, 520 - 590.

[3] Gao Z. and P.J. Antsaklis (1991). Stability of the pseudo-inverse method for reconfigurable control systems. Int. J. of Control, 53:717729.

[4] Huang R. F. and C. Y Strangel (1990). Restructurable control using proportional-integral implicit model following. J. Guidance, Control and Dynamics, 13:303-309.

[5] Maciejowski J. M. (1997). Reconfigurable Control Using Constrained Optimization, In Proc. of European Control Conference ECC'97, Brussels, Belgium, Plenary Lectures and Mini-Courses, 107 - 130.

[6] Niemann H. H. and J. Stoustrup (2002). Reliable control using the primary and dual Youla parameterization. In Proc. of 41st IEEE Conf. Decision and Control, 4353-4358.

[7] Ostroff A. J. (1985). Techniques for accommodating control effector failures on a mildly statically unstable airplane. In Proc. of the American Control Conference, 903-906.

[8] Patton R. J. (1997). Fault Tolerant Control : the 1997 situation. In Proc. of IFAC Symp. on Fault Detection Supervision and Safety of Technical Processes, Safeprocess'97, 1033-1055.

[9] M. Staroswiecki (2003), Actuator faults and the linear quadratic problem, Proc. of the 42th IEEE Conference on Decision and Control, CDC'03, Maui, Hawaii, USA, 959 - 965.

[10] M. Staroswiecki (2002), On reconfigurability with respect to actuator failures, IFAC World Congress, Barcelona, Spain.

[11] Staroswiecki M. (2005), Fault tolerant control : the pseudo-inverse method revisited, IFAC World Congress, Prague, Czech Republic.

[12] Ehrgott, M. (2005), Multicriteria Optimization, 2nd ed., 320 p., Springer, ISBN: 3-540-21398-8

[13] Ferreira, P. A.V. and Machado, M. E. S. (1996), Solving MultipleObjective Problems in the Objective Space, J. Optimization Theory and Applications, 89, 475-481. 